\title{
Effects of ribavirin on the replication and genetic stability of porcine reproductive and respiratory syndrome virus
}

\author{
Amina Khatun ${ }^{1}$, Nadeem Shabir ${ }^{1}$, Kyoung-Jin Yoon ${ }^{2}$ and Won-II Kim ${ }^{1 *}$
}

\begin{abstract}
Background: Although modified live virus (MLV) vaccines are commonly used for porcine reproductive and respiratory syndrome virus (PRRSV) control, there have been safety concerns due to the quick reversion of MLV to virulence during replication in pigs. Previous studies have demonstrated that mutant viruses emerged from lethal mutagenesis driven by antiviral mutagens and that those viruses had higher genetic stability compared to their parental strains because they acquired resistance to random mutation. Thus, this strategy was explored to stabilize the PRRSV genome in the current study.
\end{abstract}

Results: Four antiviral mutagens (ribavirin, 5-fluorouracil, 5-azacytidine, and amiloride) were evaluated for their antiviral effects against VR2332, a prototype of type 2 PRRSV. Among the mutagens, ribavirin and 5-fluorouracil had significant antiviral effects against VR2332. Consequently, VR2332 was serially passaged in MARC-145 cells in the presence of ribavirin at several concentrations to facilitate the emergence of ribavirin-resistant mutants. Two ribavirin-resistant mutants, RVRp13 and RVRp22, emerged from serial passages in the presence of 0.1 and $0.2 \mathrm{mM}$ ribavirin, respectively. The genetic stability of these resistant mutants was evaluated in MARC-145 cells and compared with VR2332. As expected, the ribavirin-resistant mutants exhibited higher genetic stability compared to their parental virus.

Conclusions: In summary, ribavirin and 5-fluorouracil effectively suppressed PRRSV replication in MARC-145 cells. However, ribavirin-resistant mutants emerged when treated with low concentrations ( $\leq 0.2 \mathrm{mM}$ ) of ribavirin, and those mutants were genetically more stable during serial passages in cell culture.

Keywords: PRRS, Ribavirin, 5-Fluorouracil, 5-Azacytidine and Amiloride

\section{Background}

Porcine Reproductive and Respiratory Syndrome (PRRS) is one of the most economically important infectious diseases of swine worldwide. The annual loss associated with PRRS to the United States swine industry has been estimated to be approximately 664 million USD [1]. PRRS virus (PRRSV), the etiological agent of PRRS, is classified as a member of the Arteriviridae virus family, along with equine arteritis virus (EAV), lactate dehydrogenase-elevating virus (LDV) of mice, and simian hemorrhagic fever virus (SHFV), and belongs to the order Nidovirales [2,3]. PRRSV is a small, enveloped virus that contains a single-stranded, non-segmented, positive-sense RNA genome approximately

\footnotetext{
* Correspondence: kwi0621@jbnu.ac.kr

${ }^{1}$ College of Veterinary Medicine, Chonbuk National University Jeonju, Korea, 664-14 Deokjin-Dong 1 Ga, Jeonju, Jeonbuk 561-756, Republic of Korea Full list of author information is available at the end of the article
}

$15 \mathrm{~kb}$ in length. The PRRSV genome encodes at least ten open reading frames (ORFs) designated as ORF1a, ORF1b, ORF2a, ORF2b, ORF3, ORF4, ORF5a, ORF5, ORF6, and ORF7 [4-8]. Because PRRSV evolves very quickly $[9,10]$, there is a great deal of genetic variability among PRRSV strains. In general, PRRSV strains are grouped into European (type 1) and North American (type 2) genotypes [11-13], but high levels of genetic variability still exist among viruses, even within the same genotype $[10,14,15]$, which results in suboptimal cross-protection between different PRRSV strains and becomes a significant impediment to the development of effective vaccines. Modified live virus (MLV) vaccines have been most commonly used to control PRRSV because they confer better protection against homologous virus strains compared to inactivated or recombinant vaccines [16]. However, there have been 
serious safety concerns about using these MLV vaccines because they quickly revert to virulence during serial passages in pigs [17-21]. Therefore, it is important to develop a new strategy to stabilize the PRRSV genome during virus replication for the purpose of vaccine safety.

Previously, a number of nucleoside analogs, including ribavirin (guanosine analog) [22,23], 5-fluorouracil (pyrimidine analog) [24], and 5-azacytidine (cytidine analog) [25], have been shown to be mutagenic, antiviral compounds that are effective against various RNA viruses, such as foot-and-mouth disease virus (FMDV), poliovirus, and hepatitis $\mathrm{C}$ virus ( $\mathrm{HCV})$. These mutagens increase the mutation frequency of RNA viruses above a tolerable error threshold during replication, ultimately driving viral infection into extinction [26-35]. Amiloride hydrochloride hydrate (hereafter "amiloride") is another antiviral drug with efficacy against many RNA viruses, including rhinovirus, coxsackievirus B3 (CVB3), coronaviruses, flaviviruses, and retroviruses [36-40]. Moreover, it has been demonstrated that amiloride increases the mutation frequency of RNA viruses, in addition to its other antiviral activities [41]. It has also been demonstrated that mutant viruses that emerged following sequential passages of $\mathrm{HCV}, \mathrm{CVB} 3$, poliovirus, and FMDV [41-44] in the presence of ribavirin had higher genetic stability than wild-type viruses. Therefore, this strategy was employed to select a mutagen-resistant strain of PRRSV that would have higher genetic stability. In the current study, the effects of four mutagens (ribavirin, 5-fluorouracil, 5-azacytidine, and amiloride) on PRRSV replication in MARC-145 cells were evaluated to select the mutagen that is most effective against PRRSV. Then, mutagen-resistant viruses were rescued after sequential passages in the presence of the mutagen and were evaluated for their genetic stability during additional sequential passages in cell culture systems.

\section{Methods}

\section{Antiviral mutagens}

Ribavirin (Sigma-Aldrich, St. Louis, MO, USA), 5fluorouracil (Sigma-Aldrich), 5-azacytidine (Sigma-Aldrich), and amiloride (Sigma-Aldrich) were used in this study. All of these mutagens were dissolved in RPMI-1640 medium (Sigma-Aldrich) at stock concentrations of $15 \mathrm{mM}$ (ribavirin) and $20 \mathrm{mM}$ (5-fluorouracil, 5-azacytidine, and amiloride), sterile-filtered using a $0.22-\mu \mathrm{m}$ syringe filter, aliquoted, and stored at $-20^{\circ} \mathrm{C}$ until use.

\section{Virus and cell culture}

VR2332, a prototype strain of PRRSV type 2, was used in the study. MARC-145, an African Green Monkey kidney cell line highly permissive to PRRSV infection, was used for PRRSV propagation and antiviral assays. MARC-145 cells were maintained in RPMI-1640 medium supplemented with heat-inactivated $10 \%$ fetal bovine serum (FBS, Invitrogen, Carlsbad, CA, USA), $2 \mathrm{mM}$ L-glutamine, and 100 $\times$ antibiotic-antimycotic solution [Anti-anti, Invitrogen; $1 \times$ solution contains $100 \mathrm{IU} / \mathrm{ml}$ penicillin, $100 \mu \mathrm{g} / \mathrm{ml}$ streptomycin, and $0.25 \mu \mathrm{g} / \mathrm{ml}$ Fungizone $^{\circ}$ (amphotericin B)] (hereafter referred to as "RPMI growth medium") at $37^{\circ} \mathrm{C}$ in a humidified $5 \% \mathrm{CO}_{2}$ atmosphere.

\section{Evaluation of effects of mutagens on PRRSV replication}

Confluent monolayers of MARC-145 cells were prepared in $25-\mathrm{cm}^{2}$ flasks and were inoculated with VR2332 at a multiplicity of infection (MOI) of 0.01. After incubation for 1 hour in a humidified $5 \% \mathrm{CO}_{2}$ incubator at $37^{\circ} \mathrm{C}$, the virus inoculum was removed, and the cell monolayer was replenished with RPMI growth medium containing one of the antiviral mutagens. Ribavirin, 5-fluorouracil, and amiloride were evaluated at six different concentrations $(0,0.2,0.4,0.6,0.8$, and $1 \mathrm{mM})$, while 5 -azactytidine was evaluated at ten different concentrations $(0,0.0001$, $0.001,0.01,0.1,0.2,0.4,0.6,0.8$, and $1 \mathrm{mM}$ ), based on previous reports $[45,46]$. The treated flasks were then incubated for four more days under the same culture conditions described above, during which time cell culture medium was collected from each flask every 24 hours, centrifuged, and stored at $-80^{\circ} \mathrm{C}$ until analysis.

\section{Virus titration assay}

Progeny virus titers were determined using a microtitration infectivity assay [47]. In brief, up to 8,10 -fold serial dilutions of samples were prepared. Confluent monolayers of MARC-145 cells prepared in 96-well plates were inoculated in quadruplicate with $100 \mu \mathrm{l}$ of each sample and were incubated for 1 hour under the same culture conditions described above. After incubation, the inoculum was discarded, and the cell monolayer was replenished with RPMI growth medium. The plates were then incubated for an additional six days and monitored for cytopathic effects (CPE) daily. The titer of each virus sample was calculated based on CPE and was expressed as a $50 \%$ tissue culture infective dose $\left(\mathrm{TCID}_{50}\right) / \mathrm{ml}[48]$.

\section{Cytotoxicity assay}

A commercially available cytotoxicity assay kit (CytoTox-Glo ${ }^{\mathrm{Tm}}$, Promega, Fitchburg, Wisconsin, USA) was used to assess the cytotoxic effects of the four antiviral mutagens in MARC-145 cells. In short, the CytoTox$\mathrm{Glo}^{\mathrm{ma}}$ assay measures a distinct protease (dead-cell protease) activity associated with cytotoxicity [49]. The assay uses a luminogenic peptide substrate (alanyl-alanylphenylalanyl-aminoluciferin; AAF-Glo ${ }^{\mathrm{rm}}$ substrate) to measure the activity of dead-cell protease released from cells that have lost membrane integrity. To determine the cytotoxicities of the mutagens, confluent monolayers of 
MARC-145 cells were prepared in $25-\mathrm{cm}^{2}$ flasks. After rinsing, the cells were replenished with RPMI growth medium containing one of the mutagens at one of four different concentrations $(0,0.5,1$, and $1.5 \mathrm{mM})$ and further incubated under the culture conditions described above. Supernatants were collected from each flask every 12 hours for up to 48 hours, and the levels of luminescence (RLU) generated from the cleavage of luminogenic AAF-Glo ${ }^{\text {th }}$ substrate by protease in the collected supernatants were measured to determine the cytotoxicity levels induced by each mutagen according to the manufacturer's instructions.

\section{Serial passages of PRRSV in MARC-145 cells in the presence of ribavirin}

VR2332 was serially passaged in cell culture in the presence of $0,0.05,0.1,0.2,0.3,0.5$, or $0.7 \mathrm{mM}$ ribavirin to study the emergence of ribavirin-resistant PRRSV mutants. Confluent monolayers of MARC-145 cells were prepared in 6-well plates and pre-treated with RPMI growth medium containing ribavirin for 7 hours prior to infection at $37^{\circ} \mathrm{C}$. After the pre-treatment incubation, cells were inoculated with VR2332 at an MOI of 0.01 . After incubation for 1 hour, virus inoculum was removed, and cells were replenished with RPMI growth medium containing the same concentrations of ribavirin as described above for post-treatment. The infection was then allowed to proceed for 24 hours, after which the cell culture fluid was collected from each well, centrifuged, and stored at $-80^{\circ} \mathrm{C}$ until use. The supernatant from each passage became the inoculum for the next passage. This procedure was repeated a total of 22 times.

Assessment of growth kinetics for ribavirin-resistant mutants in the presence of ribavirin at several concentrations using multi-step growth curve analysis

The growth competencies of two ribavirin-resistant candidate mutants (RVRp13 and RVRp22) were assessed in MARC-145 cells in the presence of ribavirin, compared to VR2332 in the presence of ribavirin. Confluent monolayers of MARC-145 cells were prepared in $25-\mathrm{cm}^{2}$ flasks, inoculated with each virus at an MOI of 0.01, and incubated for 1 hour under the same conditions described above. After incubation, the inoculum was discarded, and cells were replenished with RPMI-1640 growth medium containing several concentrations $(0$, $0.1,0.2,0.3,0.4$, and $0.5 \mathrm{mM}$ ) of ribavirin. The treated flasks were then incubated for 4 more days. Supernatants were collected from each flask every 24 hours, and the virus in these supernatants was titered.

\section{Assessment of genetic stability of ribavirin-resistant mutants during passages in MARC-145 cells}

To assess the genetic stability of the ribavirin-resistant mutants (RVRp13 and RVRp22) that arose from sequential passages of VR2332 in the presence of ribavirin as described above, the mutant viruses were passaged 10 more times, along with VR2332. Plaque purification was performed using each strain to achieve a highly homogenous virus clone, as described previously [17]. For each passage, confluent MARC-145 cell monolayer prepared in 6-well plates were inoculated with each virus strain and incubated for 1 hour. After incubation, the cells were replenished with RPMI growth medium and incubated for 24 hours. Then, supernatants were collected and used for the next passage of cells. This procedure was repeated 10 times.

Plaque purification was conducted on the supernatants collected after 10 passages to isolate 15 plaque clones per viral strain. Viral RNA was extracted from each virus clone using a commercial kit (Ribo_spin vRD ${ }^{\mathrm{m}}$, GeneAll, Seoul, South Korea) according to the manufacturer's instructions. nsp2 and ORF5, which are known to be the most variable regions in the PRRSV genome [12,14,50-54], were amplified with a one-step RT-PCR kit (Takara Bio Inc., Otsu, Shiga, Japan) and were submitted for sequencing (Macrogen Inc., South Korea). PCR amplification and sequencing primers are shown in Table 1.

\section{Data analysis}

The effects of the four mutagens on PRRSV replication were analyzed by repeated measures analysis of variance (ANOVA). The Wilcoxon signed-rank test was used to compare the mutation rates of the ribavirin-resistant mutants with that of their parental virus strain. Nucleotide sequences were aligned and analyzed using Lasergene $^{\bullet}$ MagAlign software (DNASTAR Inc., Madison, WI, USA).

\section{Results}

Effects of antiviral mutagens on in vitro PRRSV replication The effects of the four antiviral mutagens studied on PRRSV replication are summarized in Figure 1. The rate of VR2332 replication in MARC-145 cells decreased more than 100 -fold in the presence of $0.2 \mathrm{mM}$ ribavirin and was completely suppressed at concentrations of ribavirin higher than $0.2 \mathrm{mM}$. Despite the efficient antiviral effect of ribavirin at low concentrations, no significant cytotoxicity was observed, even at the highest concentration $(1.5 \mathrm{mM})$ of ribavirin studied, up to 48 hours post-treatment \{Figure 1 (A and B)\}. Similarly, 5-fluorouracil suppressed VR2332 replication in a dosedependent manner: the rate of VR2332 replication decreased approximately 100 -fold or more than 1000-fold in the presence of 0.2 or $1 \mathrm{mM} 5$-fluorouracil, respectively. No significant cytotoxicity was observed with up to $1.5 \mathrm{mM}$ 5-fluorouracil \{Figure 1 (C and D)\}. However, significant levels of cytotoxicity were observed with 1 and $1.5 \mathrm{mM}$ azacytidine at 48 hours post-treatment, 
Table 1 Sequences of primers used for PCR amplification and sequencing of the nsp2 and ORF5 regions in the VR2332 genome

\begin{tabular}{|c|c|c|c|c|}
\hline Sequenced region & Primer name & Nucleotide location $^{a}$ & Sequences $\left(5^{\prime}-3^{\prime}\right)$ & Sequenced length (bp) \\
\hline \multirow{12}{*}{ nsp2 } & ${ }_{\mathrm{p}} \mathrm{nsp2F}$ & $1249-1268$ & CCTCCTCAGAATAAGGGTTG & \multirow{12}{*}{3588} \\
\hline & ${ }_{p} n s p 2 R$ & $5120-5138$ & TGTCAAGGGCAGGGTAAG & \\
\hline & 1a $1481 R$ & $1463-1481$ & GGGAGTAGTGTTTGAGGTG & \\
\hline & 1a $1366 \mathrm{~F}$ & $1366-1383$ & CTCTTGTGCGACTGCTAC & \\
\hline & 1a $2115 R$ & $2097-2115$ & TACAGGTCAATCTITGCTG & \\
\hline & 1a $2058 \mathrm{~F}$ & $2058-2075$ & CCCAGAACAAAACCAACC & \\
\hline & 1a $2867 R$ & $2850-2867$ & ATTGCGGTGAGGACACAA & \\
\hline & 1a $2771 \mathrm{~F}$ & $2771-2788$ & TGGGAAGATTTGGCTGTT & \\
\hline & 1a $3581 R$ & $3563-3581$ & CAATGGTAAGGTCGCTCTC & \\
\hline & 1a $3511 \mathrm{~F}$ & $3511-3529$ & TCCGTGTGAGTTTGTGATG & \\
\hline & 1a $4276 R$ & $4258-4276$ & CAGTAACCTGCCAAGAATG & \\
\hline & 1a $4141 \mathrm{~F}$ & $4141-4158$ & CGCTGCTTGTGAGTITGA & \\
\hline \multirow{2}{*}{ ORF5 } & ${ }_{\mathrm{p}} \mathrm{P} 5 \mathrm{~F}^{\mathrm{r}}$ & 13716-13734 & CCTGAGACCATGAGGTGGG & \multirow{2}{*}{603} \\
\hline & ${ }_{p}$ P5 $R^{r}$ & 14457-14479 & TITAGGGCATATATCATCACTGG & \\
\hline
\end{tabular}

a: Location of primers in the full-length VR2332 genome (GenBank accession [AY150564]). p: primers used for PCR amplification and sequencing. The remaining primers were used only for sequencing. $r$ : reference primers used in a previous study [17].

although VR2332 replication was significantly suppressed when at least $0.1 \mathrm{mM} 5$-azacytidine was included in the culture medium \{Figure 1 (E and F)\}. No significant antiviral activity against VR2332 was measured at low concentrations $(<0.1 \mathrm{mM})$ of 5 -azacytidine (data not shown). Similarly, high concentrations (1 and $1.5 \mathrm{mM}$ ) of amiloride also caused significant cytotoxicity in MARC-145 cells after 36 hours post-treatment \{Figure $1(\mathrm{H})$; ; however, VR2332 replication was suppressed by amiloride in a dose-dependent manner and was completely suppressed by more than $0.6 \mathrm{mM}$ amiloride \{Figure $1(\mathrm{G})$ \}. Based on these results, ribavirin was selected for further experiments because it was most effective at suppressing PRRSV replication without causing significant cytotoxicity in MARC-145 cells.

\section{Emergence of ribavirin-resistant mutants after serial passage of PRRSV in MARC-145 cells in the presence of ribavirin}

VR2332 was serially passaged in MARC-145 cells in the presence of ribavirin at concentrations of $0,0.05,0.1$, $0.2,0.3,0.5$, and $0.7 \mathrm{mM}$. Although $0.05 \mathrm{mM}$ ribavirin failed to mediate significant suppression of VR2332 replication, concentrations of 0.1 and $0.2 \mathrm{mM}$ ribavirin were able to suppress virus replication to undetectable levels based on a virus titration assay at passage 2. However, virus replication started to resume at passages 5 and 17 in the presence of 0.1 and $0.2 \mathrm{mM}$ ribavirin, respectively. The reemerging viruses maintained increasing progeny virus production in successive passages, and two virus strains, RVRp13 and RVRp22, were recovered at passages 13 and 22 in the presence of 0.1 and $0.2 \mathrm{mM}$ ribavirin, respectively. Ribavirin doses greater than or equal to $0.3 \mathrm{mM}$ completely suppressed the replication of VR2332 below the detection limit of the virus titration assay for all 22 passages (Figure 2).

\section{Growth kinetics of ribavirin-resistant mutants in the presence of ribavirin at several concentrations}

RVRp13 and RVRp22 were evaluated for their resistance to ribavirin by assessing their growth competence in MARC-145 cells in the presence of several concentrations $(0,0.1,0.2,0.3,0.4$, and $0.5 \mathrm{mM})$ of ribavirin, compared to the growth competence of the parental virus, VR2332. Both mutant virus strains exhibited higher replication efficiency than did VR2332 in the presence of ribavirin (Figure 3); RVRp13 and RVRp22 both replicated over 10- or 100-times more efficiently than VR2332 in the presence of 0.1 or $0.2 \mathrm{mM}$ ribavirin, respectively. Moreover, VR2332 was unable to replicate in the presence of ribavirin at concentrations of $0.2 \mathrm{mM}$ or higher, whereas RVRp13 and RVRp22 were able to grow to a moderate extent in the presence of ribavirin at concentrations as high as $0.5 \mathrm{mM}$.

Higher genetic stability of ribavirin-resistant mutants after serial passages in MARC- 145 cells

The ribavirin-resistant mutants, RVRp13 and RVRp22, and VR2332 were plaque-purified and designated as RVRp13-p, RVRp22-p, and VR2332-p, respectively. The plaque-purified viruses were then serially passaged 10 times in MARC-145 cells without ribavirin. After 10 passages, 15 virus clones were rescued from each supernatant by plaque purification for each virus strain, and 

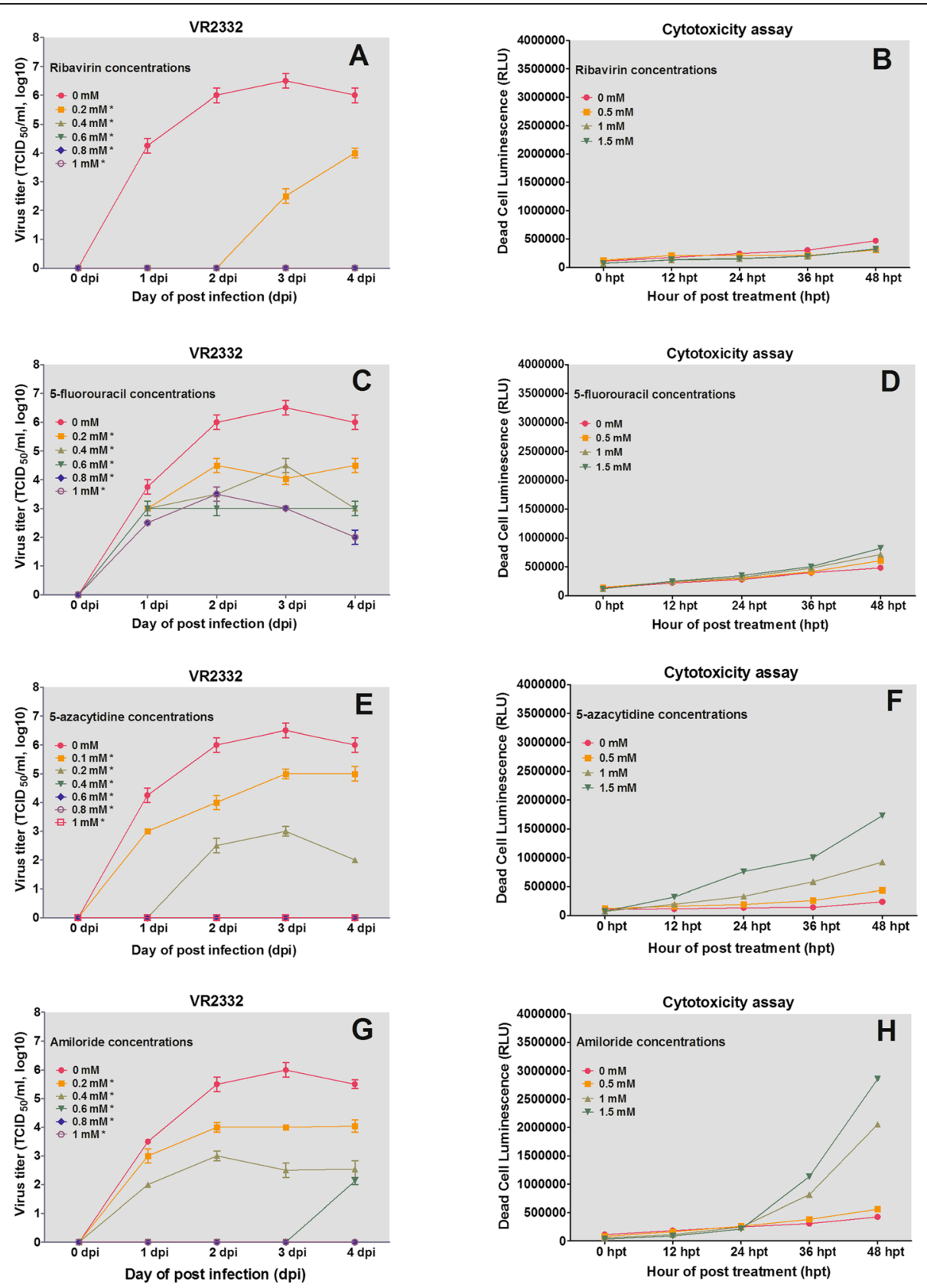

Figure 1 Evaluation of the effects of four antiviral mutagens on PRRSV replication. The effects of different concentrations of ribavirin (A), 5-fluorouracil (C), 5-azacytidine (E), and amiloride (G) in RPMI-1640 medium on the replication of the PRRSV isolate VR2332 in MARC-145 cells were evaluated, as determined by the production of progeny viruses $\left(\mathrm{TCID}_{50} / \mathrm{ml}\right)$ over time. Cytotoxicity assays were performed using cell culture fluids collected from MARC-145 cells every 12 hours after being incubated with the following mutagens: ribavirin (B), 5-fluorouracil (D), 5-azacytidine $\mathbf{( F )}$, and amiloride $\mathbf{( H )}$, as indicated in the panel. The results are expressed as the luminescence (RLU) from dead-cell protease activity. Asterisks represent a significant difference $(p<0.05)$ in virus replication after mutagen treatment compared to that after vehicle treatment.

the hypervariable regions (nsp2 and ORF5) of the 15 virus clones were amplified for sequencing. As summarized in Table 2 and Figure 4, RVRp13-p and RVRp22-p exhibited lower mutation frequencies than VR2332-p after 10 sequential passages in MARC-145 cells: $175 \mathrm{nu}-$ cleotide and 96 amino acid substitutions were identified in the nsp2 region of VR2332-p, whereas RVRp13-p had
98 nucleotide $(p<0.05)$ and 70 amino acid substitutions $(p<0.05)$ and RVRp22-p had 51 nucleotide $(p<0.001)$ and 24 amino acid substitutions $(p<0.001)$ in the same region \{Figure 4 (A)\}. In ORF5, both RVRp13-p and VR23323-p had similar mutation frequencies, with 57 nucleotide and 29 amino acid substitutions for RVRp13$\mathrm{p}$ and 51 nucleotide and 32 amino acid substitutions for 


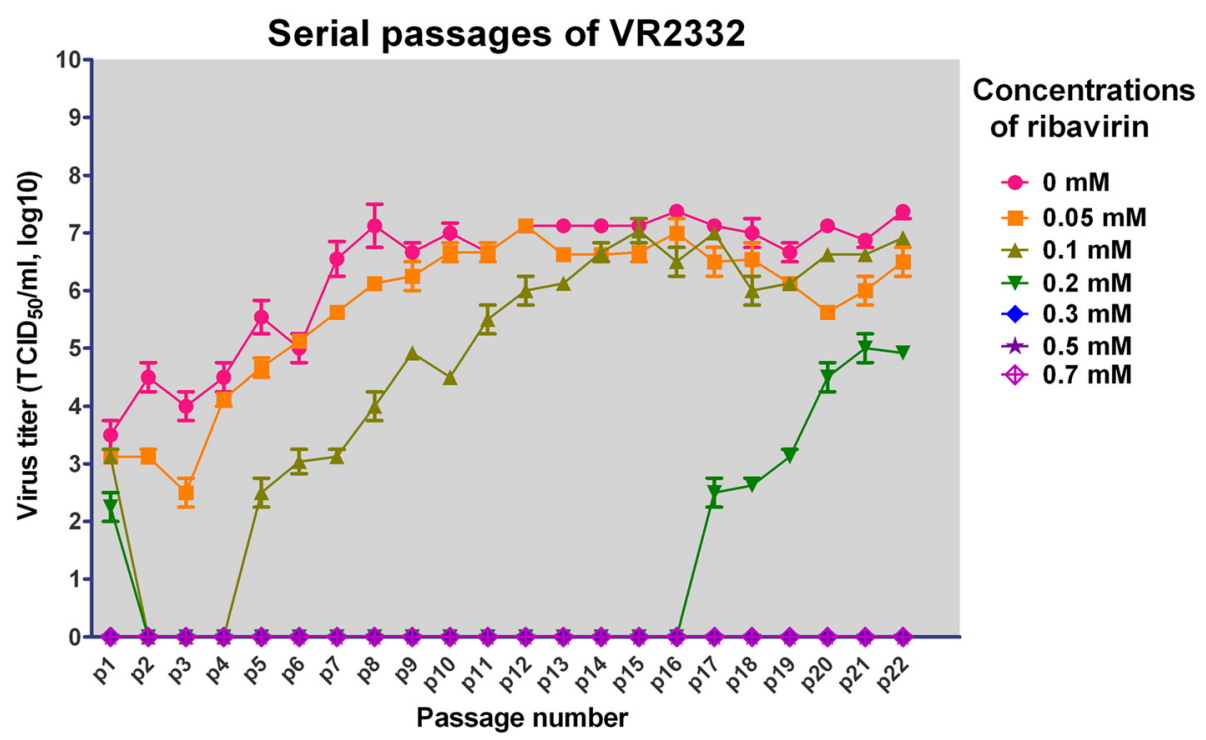

Figure 2 Emergence of ribavirin-resistant PRRSV mutants during sequential passages with ribavirin in MARC-145 cells. Ribavirin-resistant mutants emerged during serial passages of the PRRSV isolate VR2332 in MARC-145 cells in the presence of different concentrations of ribavirin, based on the presence or absence of detectable infectious progeny virus at the end of a 24-hour incubation.

VR23323-p. In contrast, RVRp22-p had a much lower $(p<0.001)$ mutation frequency, accumulating only 6 nucleotide and 4 amino acid substitutions \{Figure $4(\mathrm{~B})\}$.

\section{Discussion}

In the current study, the possibility of rescuing a genetically stable PRRSV mutant during sequential passages in MARC-145 cells in the presence of mutagens was explored on the basis of previous reports that showed that mutant viruses that emerged from lethal mutagenesis driven by antiviral mutagens exhibited higher genetic stability than wild-type viruses $[35,41,43]$. To choose the most appropriate mutagen for the study, four different antiviral mutagens (ribavirin, 5-fluorouracil, 5-azacytidine, and amiloride) were evaluated for their antiviral effects against PRRSV and for their cytotoxicity in MARC-145 cells. In the presence of ribavirin at concentrations higher than $0.2 \mathrm{mM}$, the replication of VR2332 was completely suppressed, whereas even at the highest concentration, $1.5 \mathrm{mM}$, ribavirin did not cause significant cytotoxicity in MARC-145 cells \{Figure 1 (A and B)\}. This result is in good agreement with previous studies conducted to evaluate the antiviral effects of ribavirin on several RNA viruses, including $\mathrm{HCV}$, respiratory syncytial virus (RSV), poliovirus, FMDV, and Influenza $A$ and $B$ viruses $[28,30,32,33,42,55-57]$. Recently, ribavirin was reported as a potential antiviral drug against PRRSV because it reduced virus replication approximately 100 times in PAM-pCD163 cells when added at a concentration of $0.05 \mathrm{mM}$, which was the highest concentration applied in the study [58]. However, in the current study, $0.05 \mathrm{mM}$ ribavirin failed to suppress PRRSV replication in MARC-145 cells, and concentrations higher than $0.2 \mathrm{mM}$ ribavirin were required for complete suppression of PRRSV replication (Figure 2). Unlike the results observed in PAM-pCD163 cells, no significant cytotoxicity was observed in MARC-145 cells, even when treated with $1.5 \mathrm{mM}$ ribavirin. Thus, ribavirin-resistant mutant viruses were rescued in the presence of ribavirin at concentrations as high as 0.1 and $0.2 \mathrm{mM}$. Similarly, 5-fluorouracil effectively suppressed VR2332 replication in MARC-145 cells at concentrations ranging from 0.2 to $1 \mathrm{mM}$ without causing significant cytotoxicity \{Figure $1(\mathrm{C}$ and $\mathrm{D})\}$. The effective antiviral activity of 5-fluorouracil has also been reported for rift valley fever virus, vesicular stomatitis virus, poliovirus, and FMDV [26,59-63]. In contrast, 5azacytidine or amiloride at concentrations between 1 and $1.5 \mathrm{mM}$ induced significant cytotoxicity in MARC145 cells, although concentrations of 5-azacytidine or amiloride lower than $1 \mathrm{mM}$ showed substantial antiviral efficacy against PRRSV \{Figure 1 ( $\mathrm{E}$ and $\mathrm{F}$ ) and Figure 1 $(\mathrm{G}$ and $\mathrm{H})$ \}. A previous study reported that $1 \mathrm{mM} \mathrm{5-}$ azacytidine caused significant cytotoxicity in $293 \mathrm{~T}$ and U373-MAGI ${ }_{\text {CXCR4 }}$ cells 24 hours after treatment [46] although a significant level of antiviral activity against HIV-1 was reported with 5-azacytidine at concentrations between $1 \mu \mathrm{M}$ and $100 \mu \mathrm{M}[45,46]$. However, low concentrations $(<0.1 \mathrm{mM})$ of 5 -azacytidine did not result in significant antiviral activity against PRRSV in the 


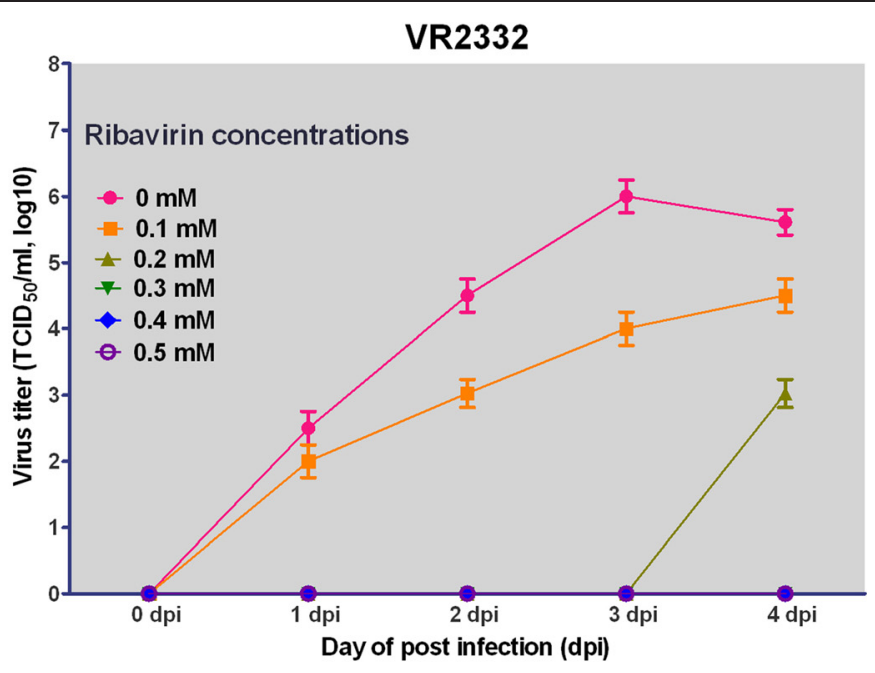

\section{RVRp13}
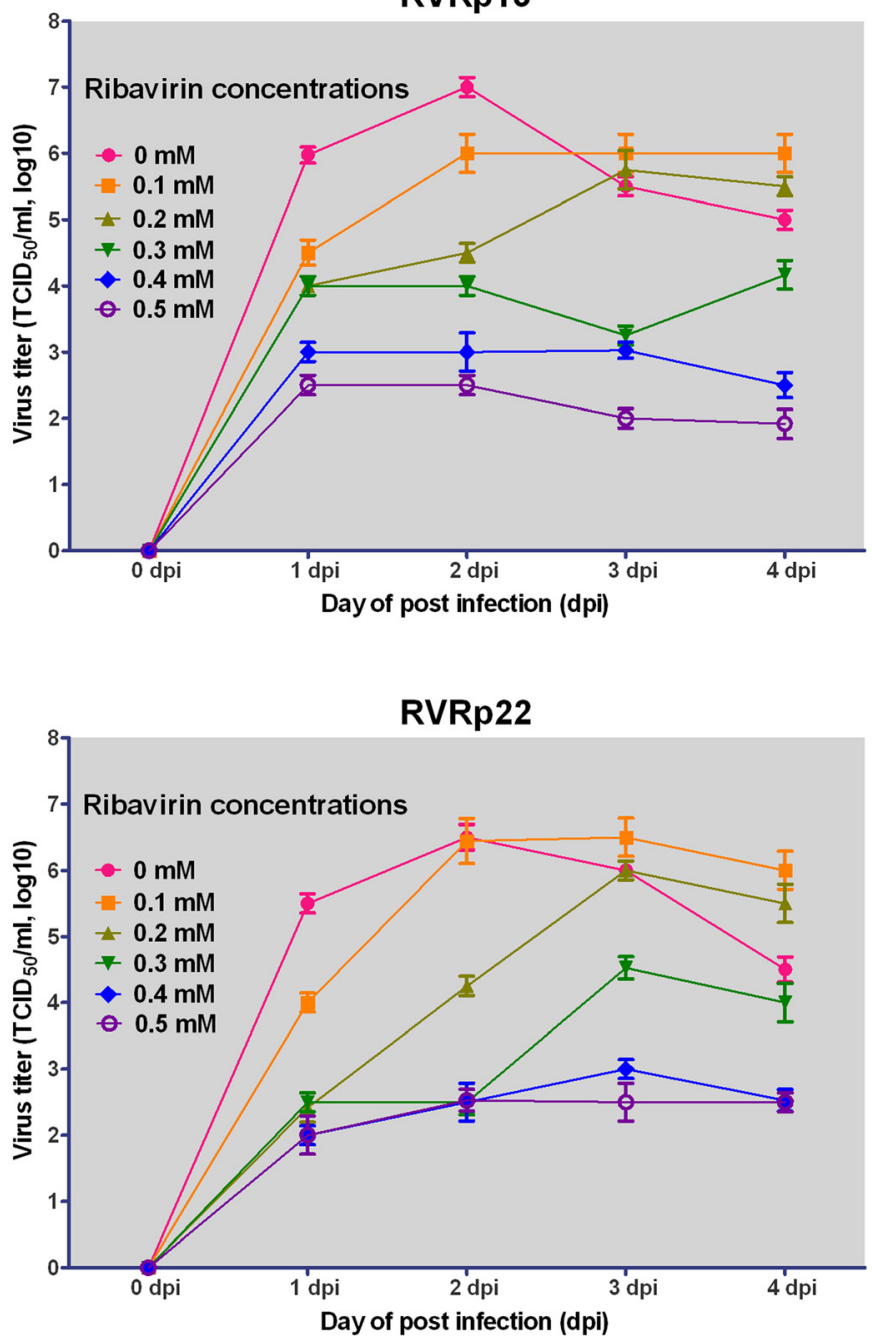

Figure 3 (See legend on next page.) 
(See figure on previous page.)

Figure 3 Assessment of the growth kinetics of ribavirin-resistant mutants in the presence of ribavirin. The growth kinetics of two ribavirin-resistant PRRSV mutants (RVRp13 and RVRp22) were evaluated in MARC-145 cells in the presence of several concentrations of ribavirin compared to their parental strain VR2332.

current study (data not shown). A previous study reported that $1 \mathrm{mM}$ amiloride was non-cytotoxic in HeLa T cells for 48 hours post-treatment [38], which conflicts with the results observed in this study showing that substantial cytotoxicity was observed with 1-1.5 mM amiloride in MARC-145 cells at 36 hours post-treatment \{Figure $1(\mathrm{H})\}$. It was speculated that this dissimilarity might be due to the different origins of the cell lines used in the studies.

Based on the initial assessment of antiviral mutagens, ribavirin was selected for further study to evaluate the possible emergence of ribavirin-resistant mutants because it showed the greatest effect on PRRSV replication without causing significant cytotoxicity even at the highest concentration (1.5 mM). Two ribavirin-resistant mutants (RVRp13 and RVRp22) were isolated at passages 13 and 22 during serial passages of VR2332 in MARC145 cells in the presence of 0.1 and $0.2 \mathrm{mM}$ ribavirin, respectively (Figure 2). Previous studies also reported the emergence of ribavirin-resistant mutants: ribavirinresistant $\mathrm{HCV}$ emerged after 7 passages in Huh7D cells cultured with $0.25 \mathrm{mM}$ ribavirin [42]. Similarly, ribavirinresistant CVB3, poliovirus, and FMDV emerged after sequential passages in HeLa or BHK-21 cells cultured with 0.05-0.8 mM ribavirin [41,43,44].

The growth competence of the ribavirin-resistant mutants RVRp13 and RVRp22 in MARC-145 cells was assessed in the presence or absence of ribavirin. Both of the ribavirin-resistant mutants showed approximately 10-100-times higher replication efficiency than VR2332 in the presence of 0.1 or $0.2 \mathrm{mM}$ ribavirin. Moreover, the replication of VR2332 was completely suppressed at concentrations higher than $0.2 \mathrm{mM}$ ribavirin, while both mutants were able to generate infectious progeny viruses, reaching titers close to $10^{3} \mathrm{TCID}_{50} / \mathrm{ml}$ in the presence of $0.5 \mathrm{mM}$ ribavirin (Figure 3). As reported in many previous studies conducted with poliovirus [43], human enterovirus 71 (HEV71) [64], FMDV [65,66], coxsackie virus B3 [41] and HCV [42], enhanced resistance of mutant viruses to ribavirin might be associated with the increased genetic fidelity that is acquired during viral passages in the presence of ribavirin. However, the higher replication efficiency might also result from virus adaptation to MARC-145 cells because the mutant viruses were rescued after 13 or 22 sequential passages. In fact, the mutant viruses replicated more efficiently in the

Table 2 Mutation frequencies of plaque-cloned, ribavirin-resistant mutants (RVRp13-P and RVRp22-P) and VR2332-p after 10 passages in MARC-145 cells

\begin{tabular}{|c|c|c|c|c|}
\hline Sequenced region & & VR2332-p & RVRp13-p & RVRp22-p \\
\hline \multirow{9}{*}{ nsp2 } & Total no. of clones sequenced ${ }^{a}$ & 15 & 15 & 15 \\
\hline & Total no. of nucleotides sequenced(3588 nt per clone) & 53820 & 53820 & 53820 \\
\hline & Total no. of mutations & 175 & 98 & 51 \\
\hline & Total no. of nucleotide deletionsin sequenced length & 63 & 0 & 6 \\
\hline & Mutation rate $/ 10^{3} \mathrm{nt}$ & 3.25 & $1.82^{*}$ & $0.94^{* *}$ \\
\hline & Total no. of amino acids sequenced(1196 aa per clone) & 17940 & 17940 & 17940 \\
\hline & Total no. of mutations & 96 & 70 & 24 \\
\hline & Total no. of amino acid deletionsin sequenced length & 21 & 0 & 2 \\
\hline & Mutation rate $/ 10^{3}$ aa & 5.35 & $3.90^{*}$ & $1.33^{* *}$ \\
\hline \multirow{7}{*}{ ORF5 } & Total no. of clones sequenced & 15 & 15 & 15 \\
\hline & Total no. of nucleotides sequenced(603 nt per clone) & 9045 & 9045 & 9045 \\
\hline & Total no. of mutations & 51 & 57 & 6 \\
\hline & Mutation rate $/ 10^{3} \mathrm{nt}$ & 5.64 & 6.30 & $0.66^{* *}$ \\
\hline & Total no. of amino acids sequenced(201 aa per clone) & 3015 & 3015 & 3015 \\
\hline & Total no. of mutations & 32 & 29 & 4 \\
\hline & Mutation rate $/ 10^{3}$ aa & 10.61 & 9.61 & $1.32^{* *}$ \\
\hline
\end{tabular}

a: The numbers of nucleotide mutations and amino acid substitutions were determined by sequencing 15 plaque-purified virus clones from cell culture fluids collected at the completion of 10 passages of each virus and comparing those samples to the original viruses not submitted to sequential cell passages. nt: nucleotide, aa: amino acid, significance levels when comparing a drug-resistant strain with VR2332 are indicated by asterisks: ${ }^{*} p<0.05$, ${ }^{* *} p<0.001$. 

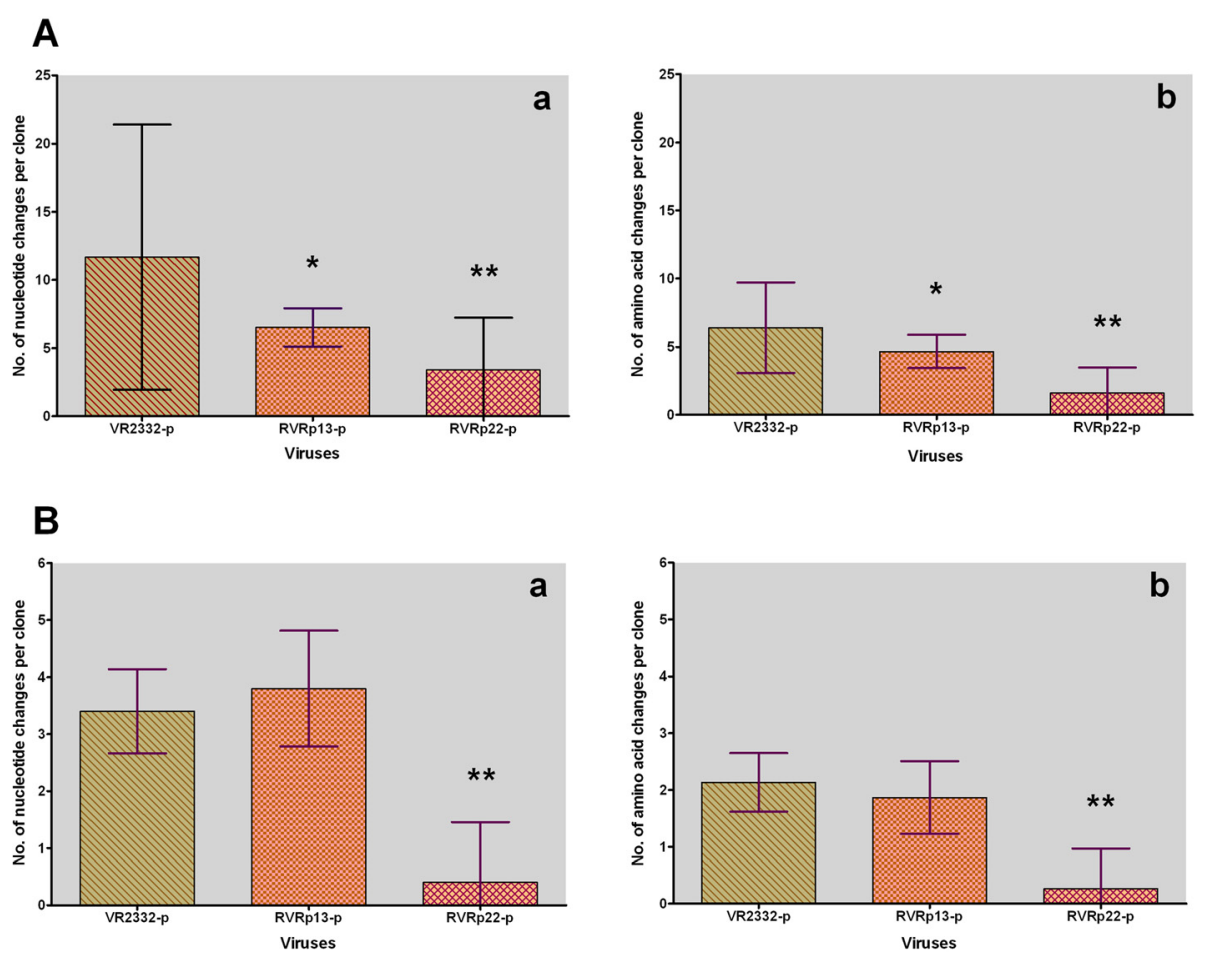

Figure 4 In vitro assessment of the genetic stability of ribavirin-resistant PRRSV mutants during serial passages in cells. The genetic stability of the plaque-purified, ribavirin-resistant mutants (RVRp13-P and RVRp22-P) was compared with that of plaque-purified VR2332 (VR2332-p) over 10 passages in MARC-145 cells. Fifteen viral clones obtained from cell culture fluids collected at the completion of 10 passages of each virus by plaque purification and nsp2 (A) and ORF5 (B) regions of each virus clone were sequenced and compared with their parental viruses (RVRp13-P, RVRp22-P, and VR2332-p) to determine nucleotide mutations (a) and amino acid (b) substitutions during the sequential passages. Asterisks represent significant differences in the mutation rate compared with VR2332 $\left.{ }^{*} p<0.05,{ }^{* *} p<0.001\right)$.

absence of ribavirin compared to VR2332. Therefore, the genetic stability of the ribavirin-resistant mutants was assessed by passaging the mutant viruses 10 times in MARC-145 cells without ribavirin in parallel with VR2332 to demonstrate that ribavirin-resistant mutants have increased genetic stability. The most variable genes (nsp2 and ORF5) in the PRRSV genome were sequenced to determine the mutation frequency of the viruses during sequential passages. RVRp13 and RVRp22 virus clones exhibited 1.8- and 3.4-fold lower numbers of nucleotide substitutions and 1.4- and 4-fold lower numbers of amino acid substitutions, respectively, in the nsp2 region compared with VR2332 virus clones \{Table 2 and Figure 4 (A)\}. In the ORF5 region, the RVRp13 virus clones exhibited mutation rates similar to those observed in VR2332 virus clones, while RVRp22 virus clones had approximately 8.5 -fold lower numbers of nucleotide substitutions and 8-fold lower numbers of amino acid substitutions compared with VR2332 virus clones \{Table 2 and Figure 4 (B)\}. Based on these results, it was concluded that the ribavirin-resistant mutants, especially RVRp22, have significantly higher genetic stability compared with their parental virus, VR2332.

\section{Conclusions}

In conclusion, ribavirin was very effective in suppressing PRRSV replication in MARC-145 cells at concentrations higher than $0.2 \mathrm{mM}$, suggesting that ribavirin could be used as a therapeutic drug against PRRSV; however, its potential usefulness against PRRSV infection remains to be confirmed in animal studies. As described in the current study, the resistant viruses emerged in the presence of low concentrations $(<0.2 \mathrm{mM})$ of ribavirin, and those resistant viruses had significantly higher genetic stability compared with VR2332. Because rapid reversion of attenuated PRRS vaccines to virulence is of great concern, RVRp22, which has a higher level of genetic stability, could be a good candidate for the development of a safer vaccine. Nonetheless, the mechanisms and genetic determinants responsible for the high genetic stability of ribavirin-resistant PRRSV should be elucidated in detail in the near future.

\section{Abbreviations}

PRRSV: Porcine reproductive and respiratory syndrome virus; MLV: Modified live virus; EAV: Equine arteritis virus; LDV: Lactate dehydrogenase-elevating virus of mice; SHFV: Simian hemorrhagic fever virus; ORF: Open reading frame; FMDV: Foot-and-mouth disease virus; HCV: Hepatitis C virus; CVB3: Coxsackievirus 
B3; FBS: Fetal bovine serum; MOl: Multiplicity of infection; CPE: Cytopathic effect; TCID: Tissue culture infective dose; AAF: Alanyl-alanylphenylalanyl-aminoluciferin RLU: Relative luminescence units; nsp2: Nonstructural protein 2, RSV, Respiratory syncytial virus; RVF: Rift valley fever virus; VSV: Vesicular stomatitis virus; HIV-1: Human immunodeficiency virus 1.

\section{Competing interests}

The authors declared that they have no competing interests.

\section{Authors' contributions}

AK performed the experiments, carried out the statistical analysis, and drafted the manuscript. NS contributed to the statistical analysis. KJY contributed to the study design and critically reviewed the manuscript. KIW conceived the study, coordinated the work described, and contributed to the manuscript preparation. All authors read and approved the final manuscript.

\section{Acknowledgments}

This research was supported by the Basic Science Research Program through the National Research Foundation of Korea (NRF), which is funded by the Ministry of Education, Science and Technology (2011-0009937) and Technology Development Program for Bio-industry (313005-3), Ministry for Food, Agriculture, Forestry and Fisheries, Republic of Korea.

\section{Author details}

${ }^{1}$ College of Veterinary Medicine, Chonbuk National University Jeonju, Korea, 664-14 Deokjin-Dong $1 \mathrm{Ga}$, Jeonju, Jeonbuk 561-756, Republic of Korea. ${ }^{2}$ Department of Veterinary Diagnostic and Production Animal Medicine, College of Veterinary Medicine, lowa State University, Ames, IA, USA.

\section{Received: 17 July 2014 Accepted: 20 January 2015}

\section{Published online: 07 February 2015}

\section{References}

1. Holtkamp DJ, Kliebenstein JB, Neumann EJ, Zimmerman JJ, Rotto HF, Yoder TK, et al. Assessment of the economic impact of porcine reproductive and respiratory syndrome virus on United States pork producers. J Swine Health Prod. 2013;21:72-84.

2. Cavanagh D. Nidovirales: A new order comprising Coronaviridae and Arteriviridae. Arch Virol. 1997;142:629-33

3. Meulenberg JJM, Hulst MM, Demeijer EJ, Moonen PLJM, Denbesten A, Dekluyver EP, et al. Lelystad virus, the causative agent of porcine epidemic abortion and respiratory syndrome (PEARS), is related to LDV and EAV. Virology. 1993;192:62-72

4. Conzelmann KK, Visser N, Vanwoensel P, Thiel HJ. Molecular characterization of porcine reproductive and respiratory syndrome virus, a member of the arterivirus group. Virology. 1993;193:329-39.

5. Firth $A E$, Zevenhoven-Dobbe JC, Wills NM, Go YY, Balasuriya UBR, Atkins $J$, et al. Discovery of a small arterivirus gene that overlaps the GP5 coding sequence and is important for virus production. J Gen Virol. 2011:92:1097-106

6. Johnson CR, Griggs TF, Gnanandarajah J, Murtaugh MP. Novel structural protein in porcine reproductive and respiratory syndrome virus encoded by an alternative ORF5 present in all arteriviruses. J Gen Virol. 2011;92:1107-16.

7. Meulenberg JJM, DenBesten AP, DKluyver E, VanNieuwstadt A, Wensvoort G Molecular characterization of Lelystad virus. Vet Microbiol. 1997;55:197-202.

8. Music N, Gagnon CA. The role of porcine reproductive and respiratory syndrome (PRRS) virus structural and non-structural proteins in virus pathogenesis. Anim Health Res Rev. 2010;11:135-63.

9. Hanada K, Suzuki Y, Nakane T, Hirose O, Gojobori T. The origin and evolution of porcine reproductive and respiratory syndrome viruses. Mol Biol Evol. 2005:22:1024-31.

10. Shi M, Lam TT, Hon CC, Hui RK, Faaberg KS, Wennblom T, et al. Molecular epidemiology of PRRSV: a phylogenetic perspective. Virus Res. 2010;154:7-17.

11. Lunney JK, Benfield DA, Rowland RR. Porcine reproductive and respiratory syndrome virus: an update on an emerging and re-emerging viral disease of swine. Virus Res. 2010;154:1-6.

12. Meng XJ, Paul PS, Halbur PG, Morozov I. Sequence comparison of open reading frames 2 to 5 of low and high virulence United States isolates of porcine reproductive and respiratory syndrome virus. J Gen Virol. 1995;76(Pt 12):3181-8.
13. Nelsen CJ, Murtaugh MP, Faaberg KS. Porcine reproductive and respiratory syndrome virus comparison: divergent evolution on two continents. J Virol. 1999;73:270-80.

14. Suarez P, Zardoya R, Martin MJ, Prieto C, Dopazo J, Solana A, et al. Phylogenetic relationships of european strains of porcine reproductive and respiratory syndrome virus (PRRSV) inferred from DNA sequences of putative ORF-5 and ORF-7 genes. Virus Res. 1996;42:159-65.

15. Yin G, Gao L, Shu X, Yang G, Guo S, Li W. Genetic diversity of the ORF5 gene of porcine reproductive and respiratory syndrome virus isolates in southwest China from 2007 to 2009. PLoS One. 2012;7:e33756.

16. Charerntantanakul W. Porcine reproductive and respiratory syndrome virus vaccines: Immunogenicity, efficacy and safety aspects. World J Virol. 2012;1:23-30.

17. Kim WI, Kim JJ, Cha SH, Yoon KJ. Different biological characteristics of wild type porcine reproductive and respiratory syndrome viruses and vaccine viruses and identification of the corresponding genetic determinants. J Clin Microbiol. 2008:46:1758-68.

18. Madsen KG, Hansen CM, Madsen ES, Strandbygaard B, Botner A, Sorensen $\mathrm{KJ}$. Sequence analysis of porcine reproductive and respiratory syndrome virus of the American type collected from Danish swine herds. Arch Virol. 1998;143:1683-700.

19. Nielsen HS, Oleksiewicz MB, Forsberg R, Stadejek T, Botner A, Storgaard T. Reversion of a live porcine reproductive and respiratory syndrome virus vaccine investigated by parallel mutations. J Gen Virol. 2001;82:1263-72.

20. Opriessnig T, Halbur PG, Yoon KJ, Pogranichniy RM, Harmon KM, Evans R, et al. Comparison of molecular and biological characteristics of a modified live porcine reproductive and respiratory syndrome virus (PRRSV) vaccine (ingelvac PRRS MLV), the parent strain of the vaccine (ATCC VR2332), ATCC VR2385, and two recent field isolates of PRRSV. J Virol. 2002;76:11837-44.

21. Storgaard T, Oleksiewicz M, Botner A. Examination of the selective pressures on a live PRRS vaccine virus. Arch Virol. 1999;144:2389-401.

22. Harris S, Robins R. Ribavirin: structure and antiviral activity relationships. In: Smith RA, Kirkpatrick W, editors. Ribavirin: A Broad Spectrum Antiviral Agent. New York: Academic; 1980. p. 1-21.

23. Wu JZ, Walker H, Lau JY, Hong Z. Activation and deactivation of a broadspectrum antiviral drug by a single enzyme: adenosine deaminase catalyzes two consecutive deamination reactions. Antimicrob Agents Chemother. 2003:47:426-31.

24. Boumah CE, Setterfield G, Kaplan JG. Purine and pyrimidine analogues irreversibly prevent passage of lymphocytes from the G1 to the $S$ phase of the cell cycle. Can J Biochem Cell Biol. 1984;62:280-7.

25. Piskala A, Sorm F. Nucleic Acids Components + Their Analogues.51. Synthesis of 1-Glycosyl Derivatives of 5-Azauracil + 5-Azacytosine. Collect Czech Chem Communications. 1964;29:2060-76.

26. Agudo R, Arias A, Domingo E. 5-fluorouracil in lethal mutagenesis of footand-mouth disease virus. Future Med Chem. 2009;1:529-39.

27. Agudo R, Ferrer-Orta C, Arias A, de la Higuera I, Perales C, Perez-Luque R, et al. A multi-step process of viral adaptation to a mutagenic nucleoside analogue by modulation of transition types leads to extinction-escape. PLOS Pathog. 2010;6:e1001072

28. Crotty S, Cameron C, Andino R. Ribavirin's antiviral mechanism of action: lethal mutagenesis? J Mol Med (Berl). 2002;80:86-95.

29. Crotty S, Cameron CE, Andino R. RNA virus error catastrophe: Direct molecular test by using ribavirin. Proc Natl Acad Sci U S A. 2001;98:6895-900.

30. Crotty S, Maag D, Arnold JJ, Zhong WD, Lau JYN, Hong Z, et al. The broadspectrum antiviral ribonucleoside ribavirin is an RNA virus mutagen. Nat Med. 2000;6:1375-9.

31. Gordon MP, Staehelin M. Studies on the Incorporation of 5-Fluorouracil into a Virus Nucleic Acid. Biochim Biophys Acta. 1959;36:351-61.

32. Gu CJ, Zheng CY, Zhang Q, Shi LL, Li Y, Qu SF. An antiviral mechanism investigated with ribavirin as an RNA virus mutagen for foot-and-mouth disease virus. J Biochem Mol Biol. 2006;39:9-15.

33. Perales C, Agudo R, Tejero H, Manrubia SC, Domingo E. Potential benefits of sequential inhibitor-mutagen treatments of RNA virus infections. PLoS Pathog. 2009;5:e1000658.

34. Sanchez-Jimenez C, Olivares I, de Avila Lucas Al, Toledano V, Gutierrez-Rivas M, Lorenzo-Redondo R, et al. Mutagen-mediated enhancement of HIV-1 replication in persistently infected cells. Virology. 2012;424:147-53.

35. Vignuzzi M, Stone JK, Arnold JJ, Cameron CE, Andino R. Quasispecies diversity determines pathogenesis through cooperative interactions in a viral population. Nature. 2006;439:344-8. 
36. Ewart GD, Mills K, Cox GB, Gage PW. Amiloride derivatives block ion channel activity and enhancement of virus-like particle budding caused by HIV-1 protein Vpu. Eur Biophys J. 2002;31:26-35.

37. Gazina EV, Harrison DN, Jefferies M, Tan H, Williams D, Anderson DA, et al. Ion transport blockers inhibit human rhinovirus 2 release. Antiviral Res. 2005;67:98-106.

38. Harrison DN, Gazina EV, Purcell DF, Anderson DA, Petrou S. Amiloride derivatives inhibit coxsackievirus B3 RNA replication. J Virol. 2008;82:1465-73.

39. Premkumar A, Wilson L, Ewart GD, Gage PW. Cation-selective ion channels formed by $\mathrm{P7}$ of hepatitis $\mathrm{C}$ virus are blocked by hexamethylene amiloride. FEBS Lett. 2004;557:99-103.

40. Wilson L, Gage P, Ewart G. Hexamethylene amiloride blocks E protein ion channels and inhibits coronavirus replication. Virology. 2006;353:294-306.

41. Levi LI, Gnadig NF, Beaucourt S, McPherson MJ, Baron B, Arnold JJ, et al. Fidelity variants of RNA dependent RNA polymerases uncover an indirect, mutagenic activity of amiloride compounds. PLoS Pathog. 2010;6:e1001163.

42. Feigelstock DA, Mihalik KB, Feinstone SM. Selection of hepatitis C virus resistant to ribavirin. Virol J. 2011;8:402.

43. Pfeiffer JK, Kirkegaard K. A single mutation in poliovirus RNA-dependent RNA polymerase confers resistance to mutagenic nucleotide analogs via increased fidelity. Proc Natl Acad Sci U S A. 2003:100:7289-94.

44. Sierra M, Airaksinen A, Gonzalez-Lopez C, Agudo R, Arias A, Domingo E. Foot-and-mouth disease virus mutant with decreased sensitivity to ribavirin: implications for error catastrophe. J Virol. 2007;81:2012-24.

45. Bouchard J, Walker MC, Leclerc JM, Lapointe N, Beaulieu R, Thibodeau L. 5azacytidine and 5-azadeoxycytidine inhibit human immunodeficiency virus type 1 replication in vitro. Antimicrob Agents Chemother. 1990;34:206-9.

46. Dapp MJ, Clouser CL, Patterson S, Mansky LM. 5-Azacytidine Can Induce Lethal Mutagenesis in Human Immunodeficiency Virus Type 1. J Virol. 2009;83:11950-8

47. Greig A. The use of a microtitration technique for the routine assay of African swine fever virus. Brief Report Arch Virol. 1975:47:287-9.

48. Reed $L$, Muench $H$. A simple method of estimating fifty percent endpoints. American J Hygiene. 1938;27:493-7.

49. Niles AL, Moravec RA, Eric Hesselberth P, Scurria MA, Daily WJ, Riss TL. A homogeneous assay to measure live and dead cells in the same sample by detecting different protease markers. Anal Biochem. 2007;366:197-206.

50. Han J, Liu G, Wang Y, Faaberg KS. Identification of nonessential regions of the nsp2 replicase protein of porcine reproductive and respiratory syndrome virus strain VR-2332 for replication in cell culture. J Virol. 2007;81:9878-90.

51. Han J, Wang Y, Faaberg KS. Complete genome analysis of RFLP 184 isolates of porcine reproductive and respiratory syndrome virus. Virus Res. 2006;122:175-82

52. Mardassi H, Mounir S, Dea S. Molecular analysis of the ORFs 3 to 7 of porcine reproductive and respiratory syndrome virus, Quebec reference strain. Arch Virol. 1995;140:1405-18

53. Nam E, Park CK, Kim SH, Joo YS, Yeo SG, Lee C. Complete genomic characterization of a European type 1 porcine reproductive and respiratory syndrome virus isolate in Korea. Arch Virol. 2009;154:629-38.

54. Tian K, Yu X, Zhao T, Feng Y, Cao Z, Wang C, et al. Emergence of fatal PRRSV variants: unparalleled outbreaks of atypical PRRS in China and molecular dissection of the unique hallmark. PLoS One. 2007;2:e526.

55. Hruska JF, Bernstein JM, Douglas Jr RG, Hall CB. Effects of ribavirin on respiratory syncytial virus in vitro. Antimicrob Agents Chemother. 1980;17:770-5

56. Oxford JS. Inhibition of the replication of influenza $A$ and $B$ viruses by a nucleoside analogue (ribavirin). J Gen Virol. 1975;28:409-14.

57. Sierra S, Davila M, Lowenstein PR, Domingo E. Response of foot-and-mouth disease virus to increased mutagenesis: influence of viral load and fitness in loss of infectivity. J Virol. 2000;74:8316-23.

58. Kim Y, Lee C. Ribavirin efficiently suppresses porcine nidovirus replication. Virus Res. 2013;171:44-53.

59. Caplen H, Peters CJ, Bishop DHL. Mutagen-directed attenuation of Rift-Valley fever virus as a method for vaccine development. J Gen Virol. 1985;66:2271-7.

60. Holland JJ, Domingo E, Delatorre JC, Steinhauer DA. Mutation frequencies at defined single codon sites in vesicular stomatitis-virus and poliovirus can be increased only slightly by chemical mutagenesis. J Virol. 1990;64:3960-2.

61. Lee CH, Gilbertson DL, Novella IS, Huerta R, Domingo E, Holland JJ. Negative effects of chemical mutagenesis on the adaptive behavior of vesicular stomatitis virus. J Virol. 1997;71:3636-40.
62. Perales $C$, Agudo R, Domingo E. Counteracting quasispecies adaptability: extinction of a ribavirin-resistant virus mutant by an alternative mutagenic treatment. PLoS One. 2009;4:e5554.

63. Pringle CR. Genetic characteristics of conditional lethal mutants of vesicular stomatitis virus induced by 5-fluorouracil, 5-azacytidine, and ethyl methane sulfonate. J Virol. 1970:5:559-67.

64. Sadeghipour S, Bek EJ, McMinn PC. Ribavirin-resistant mutants of human enterovirus 71 express a high replication fidelity phenotype during growth in cell culture. J Virol. 2013;87:1759-69.

65. Zeng J, Wang H, Xie X, Yang D, Zhou G, Yu L. An increased replication fidelity mutant of foot-and-mouth disease virus retains fitness in vitro and virulence in vivo. Antiviral Res. 2013;100:1-7.

66. Zeng J, Wang H, Xie X, Li C, Zhou G, Yang D, et al. Ribavirin-resistant variants of foot-and-mouth disease virus: the effect of restricted quasispecies diversity on viral virulence. J Virol. 2014;88:4008-20.

\section{Submit your next manuscript to BioMed Central and take full advantage of:}

- Convenient online submission

- Thorough peer review

- No space constraints or color figure charges

- Immediate publication on acceptance

- Inclusion in PubMed, CAS, Scopus and Google Scholar

- Research which is freely available for redistribution 